Eponyms in medicine revisited

\title{
The Mirizzi syndrome
}

\author{
M Pemberton, AD Wells
}

\begin{abstract}
Summary
The Mirizzi syndrome is an unusual presentation of gallstones which occurs when a gallstone becomes impacted in either Hartmann's pouch of the gallbladder or the cystic duct, causing obstruction of the common hepatic duct by extrinsic compression. The diagnosis of this syndrome is of importance because surgery in its presence is associated with an increased incidence of bile duct injury. The pathology, clinical presentation and management of this syndrome are discussed, and several illustrative case reports presented.
\end{abstract}

Keywords: Mirizzi syndrome, extrahepatic biliary obstruction

\section{Causes of extrahepatic biliary} obstruction

- intraduct: gallstones, foreign body, eg, broken T-tube, parasites (hydatid, liver fluke, roundworms)

- duct wall: congenital atresia, traumatic stricture, sclerosing cholangitis, tumour of bile duct, carcinoma of head of pancreas

- outside duct: carcinoma of head of pancreas, carcinoma of the papilla, pancreatitis, porta hepatitis metastases, chronic duodenal ulcer

Box 1

Peterborough District Hospital, Thorpe Road, Peterborough, UK M Pemberton

AD Wells

Correspondence to $\mathrm{Mr} \mathrm{M}$ Pemberton, Department of Surgery, Leicester Royal Infirmary, Leicester LE2 7LX, UK
An unusual presentation of gallstones occurs when a calculus, impacted in either Hartmann's pouch of the gallbladder or the cystic duct, causes obstruction of the common hepatic duct by extrinsic compression, a phenomenon known as the Mirizzi syndrome. ${ }^{1}$ This syndrome, which may occur in $0.7-1.4 \%$ of patients undergoing cholecystectomy, ${ }^{2-4}$ is of particular importance because surgery in its presence is associated with an increased incidence of bile duct injury when a standard cholecystectomy technique is used $^{5}$; indeed the syndrome has been cited as a trap in the surgery of gallstones. ${ }^{6}$ It is therefore very important that the diagnosis should be considered in any patient with a history of obstructive jaundice who is being prepared for surgery, and the condition emphasises the value of accurately establishing the anatomical abnormality in such cases pre-operatively (box 1). This article reviews the pathology, clinical presentation and management of this rare syndrome of extrahepatic obstruction.

\section{Pathology}

The Mirizzi syndrome develops as a result of the acute and chronic inflammatory processes that follow the impaction of either a large gallstone or multiple small gallstones in Hartmann's pouch of the gallbladder or the cystic duct in close anatomic proximity to the common hepatic duct. ${ }^{6,7}$ The presence of a long parallel cystic duct or a low insertion into the common bile duct appears to predispose the development of this syndrome, although they do not appear to be a requirement. ${ }^{4,8,9}$ As the gallbladder becomes shrunken as a result of chronic inflammation, and tends to become partially fused to the hepatic duct, the hepatic duct is itself progressively obstructed, both by the development of an inflammatory stricture and by a direct effect of the stone itself, which finally causes pressure necrosis of the intervening walls, and results in the formation of a fistula. ${ }^{4,6,7}$

The syndrome may present clinically at a variable stage of this pathological sequence of events and has been subclassified accordingly (figure 1). The original classification of McSherry, based on findings at endoscopic retrograde cholangiopancreatography (ERCP), ${ }^{10}$ divided the Mirizzi syndrome into two groups: type I, characterised by simple external compression of the common hepatic duct, and type II, defined by the presence of a cholecyst-choledochal fistula. This classification has since been further expanded to take into account the presence or obliteration of the cystic duct (IA and IB, respectively ${ }^{11,12}$ ) and the size of the defect in the common hepatic duct (III and IV) ${ }^{14}$; it is used as a guide to surgical strategy in an individual case. In the largest series reported ${ }^{4}$ (219 patients), $11 \%$ presented with a type I lesion, $41 \%$ with a type II lesion, $44 \%$ with a type III lesion, and $4 \%$ with a type IV lesion.

\section{Clinical features}

Although the Mirizzi syndrome predictably occurs in the elderly, it may occur in any patient with gallstones. ${ }^{4}$ Patients with the Mirizzi syndrome classically present with either painless jaundice or with cholangitis consequent upon biliary obstruction (boxes $2-4$ ). Nonetheless, Curet et al have recently reported a series in which only 10 out of 17 patients found at surgery to have the Mirizzi syndrome had presented with a recent history of jaundice, with five out of these 17 patients in fact having normal pre-operative liver function serum biochemistry. ${ }^{12}$ These findings supported earlier observations that a significant number of patients are not jaundiced at presentation. ${ }^{5,10,13}$ Furthermore, whilst patients may present with a history of right upper quadrant pain, ${ }^{4}$ there is little evidence to support a widely held view that the Mirizzi syndrome is more likely to occur in patients with long-standing biliary symptoms. In fact, apart from the presence or history of jaundice, there are generally few clues pointing to the specific diagnosis of the Mirizzi syndrome; a significant proportion of patients may present acutely with cholecystitis or pancreatitis. Occasionally patients 


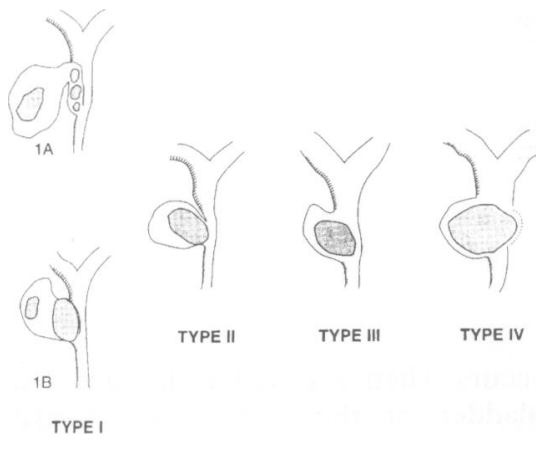

Figure 1 Classification of the Mirizzi syndrome. In type I lesions, no fistula is present a long parallel cystic duct packed with stones may be present (1A); alternatively the duct may be partly obliterated by a stone impacted within Hartmann's pouch (1B). In types II to IV a fistula into the bile duct is present, with an increasing duct defect: type II $<33 \%$; type III $3366 \%$, and type IV $>66 \%$ of bile duct circumference (after Csendes et $a l^{4}$ )

Case report
An obese 65 -year-old woman
presented with a nine week history of
abdominal pain, intermittent rigors
and jaundice. An ultrasound scan
indicated a common bile duct stone.
Following a failed ERCP the patient
developed cholangitis and required
urgent laparotomy. A shrunken gall-
bladder with dense periportal
inflammatory adhesions was found; on
attempted retrograde dissection a
stone emerged from of the bile duct
leaving a $20-30 \%$ defect, with apparent
disruption of the right hepatic duct.
The gallbladder remnant was excised,
a t-tube placed in the common bile
duct defect and the right hepatic duct
was separately cannulated. After a
stormy course the patient was
discharged from hospital five months
later. There was no dilatation of right
intrahepatic ducts on serial ultrasound
examination suggesting the opening up
of intrahepatic channels. The patient
remained well with improving liver
function biochemistry over the
following year.

Box 2 present with jaundice and a right upper quadrant mass and may be thought to have carcinoma.

\section{Diagnostic investigations}

Because of the surgical implications of the Mirizzi syndrome, it is particularly important that the biliary anatomy is adequately defined before surgery. ${ }^{14}$ Typically, one large stone is demonstrated by ultrasound, ${ }^{15,16}$ but the Mirizzi syndrome can also occur with multiple small impacted stones ${ }^{4,6,10} \mathrm{~A}$ contracted gallbladder with dilated intrahepatic and common hepatic ducts but normal calibre common bile duct are the classical sonographic findings; similar signs may be seen at CT. However, periductal inflammation may present as a mass lesion on imaging studies and be misinterpreted as carcinoma of the gall bladder, cholangiocarcinoma or metastases. ${ }^{15,16}$ Moreover these noninvasive modalities may also incorrectly indicate the problem to be a common duct stone (box 2). ERCP is a more effective way of defining anatomy of the biliary tree when the diagnosis is suspected (figure 2 ), ${ }^{17}$ and probably represents the gold standard investigation ${ }^{4,5,10,13,15}$; it also provides an opportunity to achieve relief of biliary obstruction through insertion of a stent which may actually improve the outcome of surgery, ${ }^{18,19}$ although this can be extremely difficult in the presence of tight strictures. The occasional failure of ERCP to demonstrate the presence of a fistula in type II cases ${ }^{10,12}$ emphasises the importance of routine per-operative cholangiography where Mirizzi syndrome is diagnosed. If ERCP is ineffective, then a percutaneous transhepatic cholangiogram (PTC) should be undertaken..$^{20-22}$ The typical findings in Mirizzi syndrome are a smooth lateral extrinsic narrowing of the common hepatic duct with a visible calculus in the expected position of the cystic duct (figure 3 ). There is invariably a degree of proximal narrowing with a normal-calibre distal common bile duct.

\section{Management}

The treatment of choice for this condition is surgical. Surgical strategy is determined primarily on whether or not a fistula is present. Most importantly, conventional retrograde dissection is contraindicated in favour of a fundus first approach $^{5}$ to avoid the risks involved in dissection of Calot's triangle (box 2). If the fundus is opened and excised, the stone or stones can be directly removed. A rush of bile at this stage indicates the presence of a biliary fistula and intraoperative cholangiography should then be undertaken via a foley catheter to demonstrate the anatomy fully. The gallbladder is frequently thickened as well as shrunken and frozen section to exclude carcinoma of the gallbladder may be necessary. ${ }^{23}$

For type I Mirizzi the minimum necessary surgery is done. If the cystic duct is present and no common duct stones are demonstrated on per-operative cholangiography, stones are milked back into the gallbladder and the cystic duct

\section{Case report}

A 67-year-old man presented with a one week history of abdominal pain, vomiting, rigors and jaundice.

Ultrasound and CT indicated a dilated intrahepatic tree with a stone in a dilated common bile duct; ERCP was unsuccessful. At operation, multiple omental adhesions to the porta hepatis were found with a shrunken gall and a large ductal stone. On further dissection, a biliary leak at the level of the expected cystic duct insertion. The stone was extracted and the gallbladder remnant excised; the bile duct defect was primarily closed over a t-tube. A persistent biliary leak was successfully treated with a stent placed using a combined transhepatic/retrograde approach. Nine months later he was well with normal liver function biochemistry.

\section{Case report}

A 70-year-old woman presented with four week history of abdominal pain, vomiting, rigors and jaundice. An ultrasound scan showed a dilated intrahepatic tree, and a probable stone in a dilated common bile duct. ERCP (figure 2) and PTC (figure 3) demonstrated extrinsic compression of common hepatic duct. At operation, a peri-portal inflammatory mass was found in which Calot's triangle could undefineable. The fundus of the gall bladder was opened and a stone removed. A further stone impacted in Hartmann's pouch was milked back into the gallbladder with a gush of bile. A partial cholecystectomy was performed and the stump of the gallbladder closed. A t-tube left in situ was removed after two months. One year later the patient was asymptomatic with normal liver function biochemically. 


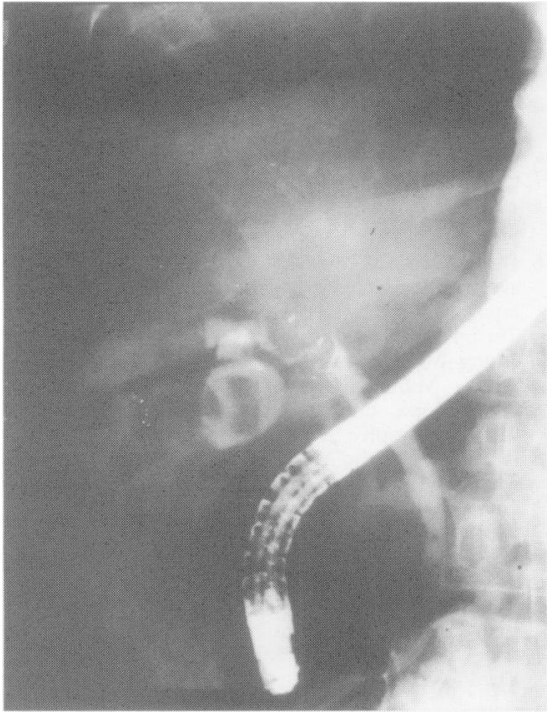

Figure 2 ERCP picture from patient in box 4 showing normal calibre distal common bile duct with obstuction of the duct midway up. The gallbladder partially fills indicating the presence of a fistula (Mirizzi II)

\section{Learning points}

- the Mirizzi syndrome is a rare cause of extrahepatic biliary obstruction caused by gallstone impaction in either Hartmann's pouch or the cystic duct

- conventional gallbladder surgery in the presence of unsuspected Mirizzi syndrome is associated with an increased risk of serious bile duct injury

- pre-operative diagnosis is of great importance and investigations should include either ERCP or PTC in order to define anatomy

- the choice of surgery is determined by whether or not a fistula into the bile duct is present, but should be via a fundus first approach, with dissection of Calot's triangle contraindicated

Box 5

1 Mirizzi PL. Syndrome del conducto hepatico. $\mathcal{f}$ Int Chir 1948; 8: $731-7$.

2 Blumgart LH. Surgery of the liver and biliary tree. Edinburgh: Churchill Livingstone, 1988.

3 Starling JR, Matallana RH. Benign mechanical obstruction of the common hepaic duct (Mirizzi syndrome). Surgery 1980; 88: 737-40.

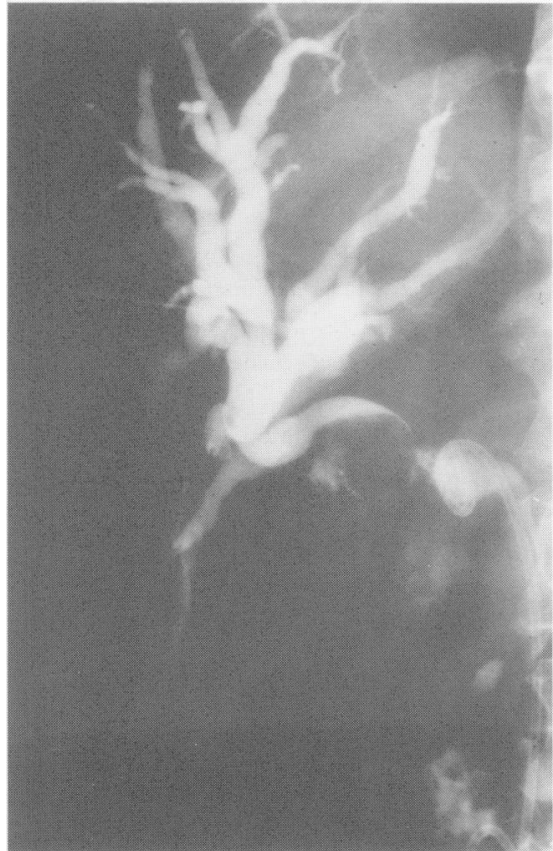

Figure 3 PTC performed in patient in box 4 after ERCP. Note the failure of a double $j-$ stent placed at time of ERCP to decompress a dilated proximal duct sytem which is caused by obstruction of the common hepatic duct which was not crossed by the stent

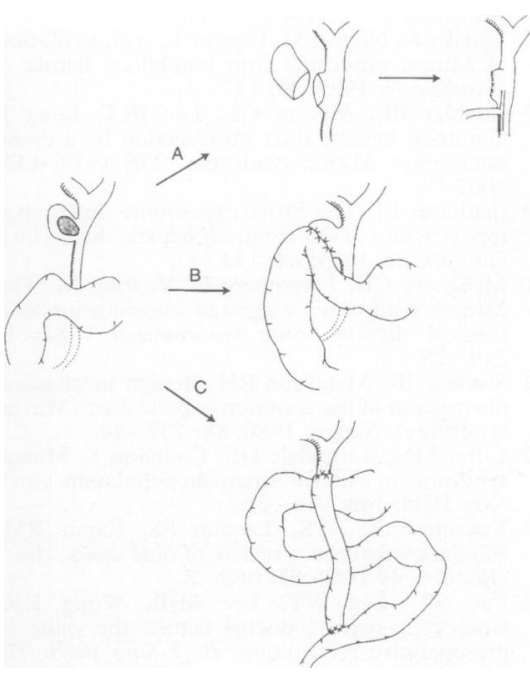
Mirizzi syndrome. A subtotal cholecystectomy with bile duct drainage via a t-tube may be performed (A) whilst in the presence of larger defects either a cholecystoduodenostomy (B) or Roux-en-Y jejunostomy (C) provide better drainage with reduced risk of long-term duct stenosis
Figure 4 Operative strategy in type II - IV
4 Csendes A, Diaz JC, Burdiles P, Maluenda F, Nava D. Mirizzi syndrome and cholecystoSurg 1989; 76: $1139-43$.
5 Baer HU, Batthews JH, Schweizer WP, Gertsch P, Blumgart LH. Management of the Mirizzi syndrome and the implications of cholecystch-
oledchal fistula. Br $\mathcal{F}$ Surg 1992; 77: 743-5. is oversewn; if it is not, a partial cholecystectomy, leaving a neck of gallbladder, unnecessary, and bile duct stenosis generally resolves as the inflammatory ensides after partial cholecystectomy. it should address the issue of the immediate anatomical defect with due follow the type primarily closed around a t-tube after performing cholecystectomy ${ }^{15}$ (box 3), a partial cholecystectomy leaving a t-tube in situ is a superior option ${ }^{4}$ (box 4). Alternatively, and especially where a larger (type III or IV) defect is suspected omy) bypass to close the cholecystcholedochal defect may be undertaken. ${ }^{5}$ This as the gallbladder wall itself is already damaged by the inflammatory process.

The fact that a proportion of patients with Mirizzi syndrome are not diagnos pre-operatively is a reminder of the perpetual need for vigilance in inflammatory tissue, and indicates the need for early cholangiography in order to define anatomy. ${ }^{12}$ This obviously applies to cases treated laparoscopically no than by conventional open surgery.

surgery, even after relief of biliary obstruction by the use of percutaneous or endoscopic stents, and case reports of alternative approaches have appeared in the literature. These have included the use of laparoscopic ${ }^{26,27}$ as well as endoscopic techniques: the percutaneous insertion of a self-expandable metallic and endoscopic lithotripsy ${ }^{29}$ have both been described, stone fragments may represent the more straightforward nonoperative option. ${ }^{30}$ Though successful as reported, these alternative treatments cannot presently be 
6 Corlette MB, Bismuth H. Biliobiliary fistula. Arch Surg 1975; 110: $377-83$.

7 Tanaka N, Nobori M, Furuya T, et al. Evolution Tanaka N, Nobori M, Furuya T, et al. Evolution
of Mirizzi syndrome with biliobiliary fistula. $\mathcal{F}$ Gastroenterol 1995; 30: $117-21$

8 Koehler RE, Melson GL, Lee JKT, Long J. Common hepatic duct obstrucction by a cystic duct stone: Mirizzi syndrome. $A \mathcal{F}$ 1979; 132: $1007-9$.

9 Balthazar EJ. The Mirizzi syndrome, inflammatory stricture of the common hepatic duct. $A m \mathcal{F}$ Gastroenterol 1975; 64: 144-8.

10 McSherry CK, Fertenberg H, Virshup M. The Mirizzi syndrome: suggested classification and Murgical therapy. Surg Gastroenterol 1982; 1: surgical
$219-25$.

11 Starling JR, Matallana RH. Benign mechanical obstruction of the common hepatic duct (Mirizzi syndrome). Surgery 1980; 88: $737-40$.

12 Curet MN, Rosendale DE, Congilosi S. Mirizzi syndrome in a native American population. $A m \mathcal{F}$ Surg 1994; 168: $616-21$

13 Venkatesh Rao PS, Tandon PS, Kapur BM. Biliobilary fistuala: a review of nine cases. $A m \mathcal{F}$ Gastroenterol 1988; 83: 652-7.

14 Fan ST, Lau WY, Lee MJR, Wong KK. Cholecysto-hepaticodochal fistula: the value of pre-operative recognition. Br f Surg 1985; 72: $743-4$.
15 Becker CD, Hassler H, Terrier F. Preoperative diagnosis of the Mirizzi syndrome: limitations of

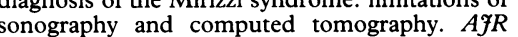
sonography and com

16 Dewar G, Chung SC, Li AK. Operative strategy in the Mirizzi syndrome. Surg Gynecol Obstet 1990; 171: $157-9$.

17 Yip AWC, Ng WS, Suen WS, Cheng CS. Type II Mirizzi syndrome: diagnosis by endoscopic retrograde cholangiopancreatography. $\mathcal{F} R$ Coll Surg Edin 1992; 37: 49-55.

18 Cotton PB. Endoscopic management of bile duct stones (apples and oranges). Gut 1984; 25 $587-97$.

19 Siegel JH, Yatto RP. Biliary endoprosthesis for the management of retained bile duct stones $A m$ the management of retained bile

20 Cruz FO, Barriga P, Tocornal J, Burhenne HJ. Radiology of the Mirizzi syndrome: diagnostic importance of the transhepatic cholangiogram. Gastrointest Radiol 1983; 8: 249-53.

21 Cornud F, Grenier P, Belghiti J, Breil P, Nahum $\mathrm{H}$. Mirizzi syndrome and biliobiliary fistulas: roentgenologic appearance. Gastrointest Radiol 1991; 6: $265-8$.

22 Ravo B, Epstin H, La Mendola S, Ger R. The Mirizzi syndrome: preoperative diagnosis by sonography and transhepatic cholangiography. Am f Gastroenterol 1986; 81: 688-90.
23 De Bakshi S, Das S, Sengupta A. Mirizzi syndrome in a patient with carcinoma of the gall bladder (letter). Br $\mathcal{F}$ Surg 1992; 79: 371.

24 Sandblom P, Tabrizian M, Rigo M, et al. Repair of common bile ducts using the gallbladder or cystic duct as a pedicled graft. Surg Gynecol Obstet 1975; 140: 425-32.

25 Yip AWC, Chow CW, Chan J, Lam KH. Mirizzi syndrome with cholecystocholedochal fistula: Pre-operative diagnosis and management. Surgery 1992; 111: $335-8$.

26 Binnie NR, Nixon SI, Palmer KR. The Mirizzi syndrome managed by endoscopic stenting and laparoscopic choloecystectomy. Br f Surg 1992; 79: 647.

27 Meng WCS, Kwok SPY, Kelly SB, Lau WY, L AKC. Management of Mirizzi syndrome by laparoscopic cholecystectomy and laparoscopic laparoscopic cholecystectomy and laparoscon

28 Adam A, Roddie ME, Benjamin IS. Case report: Mirizzi syndrome - treatment with metallic endoprosthesis. Clin Radiol 1993; 48: $198-200$.

29 Binmoeller KF, Thonke F, Soehendra N. Endoscopic treatment of Mirizzi's syndrome. Gastrointest Endosc 1993; 39: 352-6.

30 Plaiser PW, van der Hul RL, Terpstra OT, Bruning HA. Current role of extracorporeal shockwave therapy in surgery. Br F Surg 1994; 81: $174-81$.

(Sir) Alexander Fleming (1881 - 1955) was born in Ayrshire into a farming community. He qualified from St Mary's Paddington (1908) where he became assistant to Sir Almroth Wright and eventually professor of bacteriology (1928). He discovered lysozyme (1922) an antimicrobial substance present in tears, leucocytes, saliva, mucus and cartilage, and called it the body's natural antibiotic. He is immortalised for his discovery of penicillin, however, for which he shared a Nobel prize with Howard Florey and Ernst Chain (1945). He became FRS (1943) and was knighted (1944). He died of a heart attack at his home in Chelsea on 11 March 1955 and is buried in St Paul's Cathedral.

- DG Fames 